\title{
SUBSEQUENTIAL RESULTS ON UNIFORM STATISTICAL CONVERGENCE
}

\author{
TUĞBA YURDAKADIM AND LEILA MILLER-VAN WIEREN
}

Dedicated to the memory of Professor Mahmut Bajraktarević

\begin{abstract}
In this paper, we present some relationships between convergence and uniform statistical convergence of a given sequence and its subsequences. The results concerning uniform statistical convergence presented here are also closely related to earlier results regarding statistical convergence and almost convergence of sequences, and are dealing with measure and in a minor case with category. Finally, we present a Cauchy type characterization of uniform statistical convergence and a result concerning uniform statistical convergence of subseries of a series.
\end{abstract}

\section{INTRODUCTION}

The convergence of sequences has undergone numerous generalizations in order to provide deeper insights into summability theory. Convergence of sequences has different generalizations. One of the most important generalizations is uniform statistical convergence.

This type of convergence has been introduced by Brown and Freedman [4] by using uniform density and has been studied by many authors in various directions ([2], [13], [14], [17], [18]). This type of convergence is stronger than ordinary convergence so it is quite effective, especially when the classical limit does not exist.

Buck [6] has initiated the study of the relationship between the convergence of a given sequence and the summability of its subsequences. Later Agnew [1], Buck [7], Buck and Pollard [8], Miller and Orhan [16], Zeager [19] have studied this relation changing the concept of convergence. Also Dawson [9] and Fridy [12] have studied analogous results by replacing subsequences with stretching and rearrangements, respectively.

2010 Mathematics Subject Classification. 40B05, 40G15.

Key words and phrases. Uniform statistical convergence, subsequences, measure, Baire category.

Copyright (C) 2016 by ANUBIH. 
In the present paper, we are concerned with the relationships between the convergence, uniform statistical convergence of a given sequence and its subsequences in the sense of different measures. We also obtain a minor corollary by using the concept of Baire category. Finally, we give a Cauchy type criterion that characterizes uniform statistical convergence and a result on uniform statistical convergence of subseries of a given series.

Now let us recall some known notions. Let $A \subseteq \mathbb{N}$. If $n, m \in \mathbb{N}$, by $A(n, m)$ we denote the cardinality of the set of numbers $i$ in $A$ such that $n \leq i \leq m$. Numbers

$$
\underline{\mathrm{d}}(A)=\liminf _{n \rightarrow \infty} \frac{A(1, n)}{n}, \bar{d}(A)=\limsup _{n \rightarrow \infty} \frac{A(1, n)}{n}
$$

are called the lower and the upper asymptotic density of the set $A$, respectively. If $\mathrm{d}(A)=\bar{d}(A)$ then it is said that $d(A)=\mathrm{d}(A)=\bar{d}(A)$ is the asymptotic density of $A$. The uniform density of $A \subseteq \mathbb{N}$ has been introduced in [4], [5] as follows:

$$
\underline{\mathrm{u}}(A)=\lim _{n \rightarrow \infty} \frac{\min _{i \geq 0} A(i+1, i+n)}{n}, \bar{u}(A)=\lim _{n \rightarrow \infty} \frac{\max _{i \geq 0} A(i+1, i+n)}{n}
$$

are respectively called the lower and the upper uniform density of the set $A$ (the existence of these bounds is also mentioned in [2]). If $\underline{\mathrm{u}}(A)=\bar{u}(A)$, then $u(A)=\underline{\mathrm{u}}(A)$ is called the uniform density of $A$. It is clear that for each $A \subseteq \mathbb{N}$ we have

$$
\underline{\mathrm{u}}(A) \leq \underline{\mathrm{d}}(A) \leq \bar{d}(A) \leq \bar{u}(A) .
$$

The concept of statistical convergence has been introduced in [11] as follows: Let $x=\left(x_{n}\right)$ be a sequence of complex numbers. The sequence $x$ is said to be statistically convergent to a complex number $L$ provided that for every $\varepsilon>0$ we have $d\left(A_{\varepsilon}\right)=0$, where $A_{\varepsilon}=\left\{n \in \mathbb{N}:\left|x_{n}-L\right| \geq \varepsilon\right\}$. If $x=\left(x_{n}\right)$ converges statistically to $L$, then we write $s t-\lim x=L$.

Next we introduce the concept of uniform statistical convergence which is the primary topic of this paper. A sequence $x$ is said to be uniformly statistically convergent to $L$ or $I_{u}$ convergent to $L$ provided that for every $\varepsilon>0$ we have $u\left(A_{\varepsilon}\right)=0$, where $A_{\varepsilon}=\left\{n \in \mathbb{N}:\left|x_{n}-L\right| \geq \varepsilon\right\}$. If $x=\left(x_{n}\right)$ converges uniformly statistically to $L$, then we write $s t_{u}-\lim x=L$.

Miller ([15]) has shown that $S=\left\{s_{n}\right\}$ converges to $L$ statistically if and only if "most" of the subsequences of $S$ converge to $L$ statistically, by establishing a one-to-one correspondence between the interval $(0,1]$ and the collection of all subsequences of the sequence. Namely, if $x \in(0,1]$, then $x$ has a unique binary expansion

$$
x=\sum_{n=1}^{\infty} e_{n}(x) 2^{-n}, e_{n}(x) \in\{0,1\}
$$


with infinitely many ones. For each $x \in(0,1]$, let $S(x)$ denote the subsequence of $S$ obtained by the following rule: $s_{n}$ is in the subsequence if and only if $e_{n}(x)=1$. Clearly the mapping $x \rightarrow S(x)$ is a one-to-one onto mapping between $(0,1]$ and the collection of all subsequences of $S$. In [15] it is shown that $S=\left\{s_{n}\right\}$ converges to $L$ statistically if and only if the set of $x \in(0,1]$, for which $S(x)$ converges to $L$ statistically has Lebesgue measure 1 .

In a later paper [16], Orhan and Miller, have studied the concept of almost convergence of sequences and have obtained some results regarding subsequences. Namely a bounded sequence $S=\left\{s_{n}\right\}$ is almost convergent to $L$ if

$$
\lim _{n \rightarrow \infty} \frac{\sum_{i=m+1}^{m+n} s_{i}}{n}=L
$$

uniformly in $m$ (see [16] ). Orhan and Miller [16] have shown that if $S$ almost converges to $L$, then the the set of $x \in(0,1]$, for which $S(x)$ almost converges to $L$ must have measure 0 or 1 (both values may occur).

Now it is known that any bounded uniformly statistically convergent sequence is also almost convergent to the same limit (see [2]) and of course is also statistically convergent to the same limit. However even statistical and almost convergence combined do not necessarily yield uniform statistical convergence as can be seen in the following example:

Example 1. The sequence $S$ is given by

$$
\frac{1}{2}, \frac{1}{2}, 0,1, \frac{1}{2}, \frac{1}{2}, \frac{1}{2}, \frac{1}{2}, 0,1,0,1, \frac{1}{2}, \frac{1}{2}, \frac{1}{2}, \frac{1}{2}, \frac{1}{2}, \frac{1}{2}, \frac{1}{2}, \frac{1}{2}, \ldots
$$

where segments of $\frac{1}{2}$ 's of length $2^{k}, k=1,2,3,4 \ldots$ are alternated with segments $0,1,0,1 \ldots$ alternating 0 's and 1's of length $2 k, k=1,2,3,4 \ldots$. It is easy to check that this sequence both statistically and almost converges to $\frac{1}{2}$ but it is not uniformly statistically convergent.

First we look at the simple case of sequences consisting of 0's and 1's.

Lemma 1. Suppose $S=\left\{s_{n}\right\}$ is a sequence consisting of 0 's and 1 's. Then $S=\left\{s_{n}\right\}$ is uniformly statistically convergent to $0(1)$ if and only if $S=\left\{s_{n}\right\}$ is almost convergent to $0(1)$, respectively.

Proof. As we have mentioned, from [2], it is known that if a bounded sequence is uniformly statistically convergent then it is almost convergent to the same limit. Now suppose $S=\left\{s_{n}\right\}$ is almost convergent to 0 . For given 
$\varepsilon>0$, we show that $\bar{u}\left\{n:\left|s_{n}-0\right|>\varepsilon\right\}=0$. It is easy to see that

$$
\begin{aligned}
\bar{u}\left\{n:\left|s_{n}-0\right|>\varepsilon\right\} & =\lim _{n \rightarrow \infty} \max _{m \geq 0} \frac{\left|\left\{i: m+1 \leq i \leq m+n,\left|s_{i}-0\right|>\varepsilon\right\}\right|}{n} \\
& =\lim _{n \rightarrow \infty} \max _{m \geq 0} \frac{\sum_{i=m+1}^{m+n} s_{i}}{n}=0
\end{aligned}
$$

due to the almost convergence of $S=\left\{s_{n}\right\}$ to 0 . Hence $S$ is uniformly statistically convergent to 0 . Note that if $S=\left\{s_{n}\right\}$ is almost convergent to 1 then the sequence $1-S$ is almost convergent to 0 and we can apply the previous argument on $1-S$.

Now we give the following theorem for uniform statistical convergence.

Theorem 1. Suppose the sequence $S=\left\{s_{n}\right\}$ converges uniformly statistically to $L$ and let

$$
X:=\{x \in(0,1]: S(x) \text { is uniformly statistically convergent to } L\} .
$$

Then $m(X)=0$ or 1 . Both values may occur for properly chosen sequences.

Proof. Since $\mathrm{X}$ is tail set, i.e. if $x \in X$ and if $x^{\prime}$ has the same digits as $x$ except for finitely many, then $x^{\prime} \in X$, $\mathrm{X}$ is nonmeasurable or $m(X)=0$ or 1 . To show $\mathrm{X}$ is measurable:

$$
\begin{aligned}
X & =\bigcap_{j \in \mathbb{N}}\left\{x \in(0,1]: \bar{u}\left(\left\{n:\left|s(x)_{n}-L\right|>\frac{1}{j}\right\}\right)=0\right\} \\
& =\bigcap_{j \in \mathbb{N}}\left\{x \in(0,1]: \lim _{n \rightarrow \infty} \max _{m \geq 0} \frac{\left|\left\{i: m+1 \leq i \leq m+n,\left|s(x)_{i}-L\right|>\frac{1}{j}\right\}\right|}{n}=0\right\} \\
& =\bigcap_{j \in \mathbb{N}} \bigcap_{k \in \mathbb{N}} \bigcup_{N \in \mathbb{N}} \bigcap_{m=1}^{\infty} \bigcap_{n=N}^{\infty}\{x \in(0,1]: \\
& \left.\frac{\left|\left\{i: m+1 \leq i \leq m+n,\left|s(x)_{i}-L\right|>\frac{1}{j}\right\}\right|}{n}<\frac{1}{k}\right\} .
\end{aligned}
$$

But each set

$$
\left\{x \in(0,1]: \frac{\left|\left\{i: m+1 \leq i \leq m+n,\left|s(x)_{i}-L\right|>\frac{1}{j}\right\}\right|}{n}<\frac{1}{k}\right\}
$$

is of the form $G \backslash M$ where $\mathrm{G}$ is an open set and M has Lebesgue measure 0 . Therefore $X$ is measurable so $m(X)=0$ or 1 . Finally we add two examples to establish that both outcomes may occur.

Example 2. Let $S$ be given by:

$$
0,1,0,0,1,0,0,0,0,1,0,0,0,0,0,0,0,0,1, \ldots
$$


where we alternate $2^{k} 0$ 's for $k=0,1,2,3 \ldots$ with single 1 's. It is easy to see that this sequence uniformly statistically converges to 0 and also all subsequences $S(x)$ for normal $x \in(0,1]$ uniformly statistically converge to 0 (normal numbers are defined as $x \in[0,1)$ for which $\frac{1}{n} \sum_{k=1}^{n} e_{k}(x) \rightarrow \frac{1}{2}$ ). Therefore in this case $m(X)=1$.

Example 3. Let $S$ be given by:

$$
\begin{aligned}
& \underbrace{0,1,0,1, \ldots, 0,1}_{n_{1}} ; \underbrace{0,0,1,0,0,1, \ldots, 0,0,1}_{n_{2}} ; \underbrace{0,0,0,1,0,0,0,1,0,0,0,1 \ldots, 0,0,0,1}_{n_{3}} ; \ldots
\end{aligned}
$$

with $n_{1}, n_{2}, n_{3}, \ldots$ as in part b) of the proof of Theorem 2.3. in [16]. Then $S$ is almost convergent and hence by Lemma 1 uniformly statistically convergent to 0 and from the same proof in [16] it satisfies $m(X)=0$.

It is not hard to prove the following well known theorem (see [8]).

Theorem 2. A sequence $S=\left\{s_{n}\right\}$ is divergent if and only if $m(Y)=1$ where $Y:=\{x \in(0,1]: S(x)$ is divergent $\}$.

From the above theorem, we conclude that if $S=\left\{s_{n}\right\}$ converges uniformly statistically to $L$, but is divergent, then $m\left(C_{L}\right)=0$ where $C_{L}:=$ $\{x \in(0,1]: S(x)$ converges to $L\}$.

Due to this example, in order to get a theorem showing that most subsequences of a statistically convergent sequence are convergent, the following measure was introduced by Miller ( $[15])$.

In the following, if $A=\left\{k_{n}\right\}$ is any subset of $\mathbb{N}, m_{A}$ will denote the unique probability measure defined on the Borel subsets of $(0,1]$ having the following property:

$$
m_{A}\left(\left\{x \in(0,1]: e_{j}(x)=1\right\}\right)= \begin{cases}\frac{1}{2}, & \text { if } j \notin A \\ \frac{1}{2^{n}}, & \text { if } j=k_{n}\end{cases}
$$

and $\left\{e_{n}(x)\right\}$ is a sequence of independent random variables with respect to $m_{A}$. (See $\left.[15]\right)$.

Using this measure, Miller (see [15]) proved that a sequence $S=\left\{s_{n}\right\}$ converges statistically to $L$ if and only if there exists a subset $A$ of $\mathbb{N}$ having statistical density zero such that $m_{A}\left(\left\{x \in(0,1]: \lim _{n \rightarrow \infty}(S(x))_{n}=L\right\}\right)=1$.

Using the definition of uniform statistical convergence, it is easy to show that if $S=\left\{s_{n}\right\}$ is a sequence such that there exists a subset $A$ of $\mathbb{N}$ having uniform density zero for which $\left\{s_{n_{k}}\right\}$ converges to $L$ (in the normal sense), 
where $\left\{n_{k}\right\}=\mathbb{N} \backslash A$, then $S=\left\{s_{n}\right\}$ converges uniformly statistically to $L$ (see [2]). The reverse implication does not hold [3].

Therefore we are able prove a theorem analogous to one part of the before mentioned Theorem 2, [15].

Theorem 3. If $S=\left\{s_{n}\right\}$ is a sequence such that there exists a subset $A$ of $\mathbb{N}$ having uniform density zero for which

$$
m_{A}\left(\left\{x \in(0,1]: \lim _{n \rightarrow \infty}(S(x))_{n}=L\right\}\right)=1
$$

for some $L$, then $S$ converges uniformly statistically to $L$.

Proof. Suppose that $S=\left\{s_{n}\right\}$ is not uniformly statistically convergent and $A$ is any subset of $\mathbb{N}$ having uniform density zero. Then by the earlier mentioned fact (from [2]) $\left\{s_{n_{k}}\right\}$, where $\left\{n_{k}\right\}=\mathbb{N} \backslash A$, does not converge. By using an argument completely analogous to the one in the proof of Theorem 2 , [15], we can verify that

$$
m_{A}(\{x \in(0,1]: S(x) \text { is convergent }\})=0 .
$$

Buck ([6], [7]) has given a characterization of convergent sequences by proving that if $S$ is a nonconvergent sequence, then no regular summability matrix can sum every subsequence of $S$. Analogues to this result have been given by Dawson [9] and Fridy [12] by replacing subsequence with streching and rearrangement, respectively. Zeager has given both statistical convergence and $A$-statistical convergence analogues to all of those results. Now we give these following simple but noteworthy results with the use of uniform statistical convergence.

Theorem 4. A complex number sequence $S$ is convergent if and only if every subsequence of $S$ is uniformly statistically convergent.

Proof. If $S$ is convergent then every subsequence of $S$ is convergent which implies it is also uniformly statistically convergent. Conversely, if every subsequence of $S$ is uniformly statistically convergent then it is statistically convergent. The proof follows from Theorem 1 of [19].

Remark 1. Similarly, we also have that a complex number sequence $S$ is convergent if and only if every rearrangement (streching) of $S$ is uniformly statistically convergent.

Corollary 1. Suppose $S=\left\{s_{n}\right\}$ is a divergent sequence. Then the set

$$
\{x \in(0,1]: S(x) \text { is uniformly statistically convergent }\}
$$

is of first category. 
Proof. Since the set $\{x \in(0,1]: S(x)$ is uniformly statistically convergent $\}$ is a subset of the set $\{x \in(0,1]: S(x)$ is statistically convergent $\}$ and the latter set is of first category ([16]), the proof follows by the fact that any subset of a first category set is a first category set.

Next we introduce a natural Cauchy type condition equivalent to uniform statistical convergence of sequences.

Definition 1. A sequence $s=\left\{s_{n}\right\}$ is said to be $I_{u}-$ Cauchy if for every $\varepsilon>0$, there exists a positive integer $N=N(\varepsilon)$ such that

$$
\bar{u}\left(\left\{n:\left|s_{n}-s_{N}\right|>\varepsilon\right\}\right)=0 .
$$

Theorem 5. The sequence $s=\left\{s_{n}\right\}$ is $I_{u}-$ Cauchy if and only if $s=\left\{s_{n}\right\}$ is $I_{u}$ convergent.

Proof. Suppose $s=\left\{s_{n}\right\}$ is $I_{u}$ convergent to $L$. Then given $\varepsilon>0$ there exists $N$ so that $\left|s_{N}-L\right|<\frac{\varepsilon}{2}$. Then for any $i$, one can see that $\left|s_{i}-s_{N}\right|>\varepsilon$ implies $\left|s_{i}-L\right|>\frac{\varepsilon}{2}$. So for any $m, n$,

$$
\left\{m+1 \leq i \leq n+1:\left|s_{i}-s_{N}\right|>\varepsilon\right\} \subseteq\left\{m+1 \leq i \leq n+1:\left|s_{i}-L\right|>\frac{\varepsilon}{2}\right\}
$$

holds and therefore

$$
\begin{aligned}
& \lim _{n \rightarrow \infty} \max _{m \geq 0} \frac{\left|\left\{i: m+1 \leq i \leq m+n,\left|s_{i}-s_{N}\right|>\varepsilon\right\}\right|}{n} \\
& \leq \lim _{n \rightarrow \infty} \max _{m \geq 0} \frac{\left|\left\{i: m+1 \leq i \leq m+n,\left|s_{i}-L\right|>\frac{\varepsilon}{2}\right\}\right|}{n}=0 .
\end{aligned}
$$

So $\bar{u}\left(\left\{n:\left|s_{n}-s_{N}\right|>\varepsilon\right\}\right)=0$, i.e., $s=\left\{s_{n}\right\}$ is $I_{u}-$ Cauchy .

Conversely assume that $s=\left\{s_{n}\right\}$ is $I_{u}-$ Cauchy. Now for $j=1,2,3, \ldots$, $n, \ldots$ take $N_{\frac{1}{j}}$ so that

$$
\bar{u}\left(\left\{n:\left|s_{n}-s_{N_{\frac{1}{j}}}\right|>\frac{1}{j}\right\}\right)=0 .
$$

It is easy to see that the subsequence $\left\{s_{N_{\frac{1}{j}}}\right\}$ must be Cauchy (in the usual sense) and hence there exists $L \in \mathbb{R}, L=\lim _{j \rightarrow \infty} s_{N_{\frac{1}{j}}}$. Now we show $s=$ $\left\{s_{n}\right\}$ is $I_{u}$ convergent to $L$. Let $\varepsilon>0$ be arbitrary, fix $j \in \mathbb{N}$ so that

$$
\left|s_{N_{\frac{1}{j}}}-L\right|<\frac{\varepsilon}{2} \text { and } \frac{1}{j}<\frac{\varepsilon}{2}
$$


Now for any $i,\left|s_{i}-L\right|>\varepsilon$ implies $\left|s_{i}-s_{N_{\frac{1}{j}}}\right|>\frac{\varepsilon}{2}$. Thus for any $m, n$,

$$
\begin{aligned}
\left\{m+1 \leq i \leq n+1:\left|s_{i}-L\right|>\varepsilon\right\} & \subseteq\left\{m+1 \leq i \leq n+1:\left|s_{i}-s_{N_{\frac{1}{j}}}\right|>\frac{\varepsilon}{2}\right\} \\
& \subseteq\left\{m+1 \leq i \leq n+1:\left|s_{i}-s_{N_{\frac{1}{j}}}\right|>\frac{1}{j}\right\}
\end{aligned}
$$

holds. Since

$$
\lim _{n \rightarrow \infty} \max _{m \geq 0} \frac{\left|\left\{m+1 \leq i \leq n+1:\left|s_{i}-s_{N_{\frac{1}{j}}}\right|>\frac{1}{j}\right\}\right|}{n}=0
$$

we conclude that

$$
\lim _{n \rightarrow \infty} \max _{m \geq 0} \frac{\left|\left\{m+1 \leq i \leq n+1:\left|s_{i}-L\right|>\varepsilon\right\}\right|}{n}=0 .
$$

Since $\varepsilon$ is arbitrary, $s=\left\{s_{n}\right\}$ is $I_{u}$ convergent to $L$.

Dindoš, Šalát and Toma [10] have studied the statistical convergence of infinite series, i.e., an infinite series $\sum_{n=1}^{\infty} a_{n}$ (with real or complex terms) is called statistically convergent to an $s(s \in \mathbb{R}$ or $s \in \mathbb{C})$ if $s t-\lim s_{n}=s$ with $s_{n}=\sum_{k=1}^{n} a_{k}(n=1,2,3, \ldots)$. By considering the unique infinite binary expansion of $x \in(0,1]$, they have associated with the number $x$ the infinite series

$$
(x):=\sum_{k=1}^{\infty} e_{k}(x) a_{k}
$$

which after omitting the zero terms can be identified with a (infinite) subseries of the series $\sum_{n=1}^{\infty} a_{n}$. Defining $C\left(\sum_{n=1}^{\infty} a_{n}\right)$ and $C_{s t a t}\left(\sum_{n=1}^{\infty} a_{n}\right)$ by the set of all numbers $x \in(0,1]$ such that the series $(x)$ converges and statistically converges, respectively, they have studied the Lebesgue measure of these sets.

Let

$C_{\text {stat }}^{\text {unif }}\left(\sum_{n=1}^{\infty} a_{n}\right)=\{x \in(0,1]:(x)$ is uniformly statistically convergent series $\}$.

Since $C\left(\sum_{n=1}^{\infty} a_{n}\right) \subset C_{\text {stat }}^{\text {unif }}\left(\sum_{n=1}^{\infty} a_{n}\right) \subset C_{\text {stat }}\left(\sum_{n=1}^{\infty} a_{n}\right)$, and considering Theorem 2.2 of [10] is useful to note the following

Remark 2. Let $\sum_{n=1}^{\infty} a_{n}$ be a convergent series. Then if $\sum_{n=1}^{\infty}\left|a_{n}\right|^{2}<\infty$ then

$$
m\left(C_{\text {stat }}^{\text {unif }}\left(\sum_{n=1}^{\infty} a_{n}\right)\right)=1
$$




\section{REFERENCES}

[1] R. P. Agnew, Summability of subsequences, Bull. Amer. Math. Soc., 50 (1944), 596598.

[2] V. Balaz and T. Salat, Uniform density $u$ and corresponding $I_{u}$ - convergence, Math. Commun., 11 2006, 1-7.

[3] P. Barbarski, R. Filipów, N. Mroz̀ek and P. Szuca, Uniform density u and $I_{u}$ - convergence on a big set, Math. Commun., 16 (2011), 125-130.

[4] T. C. Brown and A. R. Freedman, Arithmetic progressions in Lacunary sets, Rocky Mountain J. Math., 17 (1987), 587-596.

[5] T. C. Brown and A. R. Freedman, The uniform density of sets of integers and Fermat's last theorem, C. R. Math. Ref. Acad. Sci. Canada, XII (1990), 1-6.

[6] R. C. Buck, A note on subsequences, Bull. Amer. Math. Soc., 49 (1943), 924-931.

[7] R. C. Buck, An addentum to "a note on subsequences", Proc. Amer. Math. Soc., 7 (1956), 1074-1075.

[8] R. C. Buck and H. Pollard, Convergence and summability properties of subsequences, Bull. Amer. Math. Soc., 49 (1943), 924-931.

[9] D. F. Dawson, Summability of subsequences and strechings of sequences, Pacific J. Math., 44 (1973), 455-460.

[10] M. Dindoš, T. Šalát and V. Toma, Statistical convergence of infinite series, Czechoslovak Math. J., 53 (2003), 989-1000.

[11] H. Fast, Sur la convergence statistique, Colloq. Math., 2 (1951), 241-244.

[12] J. A. Fridy, Summability of rearrangements of sequences, Math. Z., 143 (1975), 187192.

[13] R. G. Antonini and G. Grekos, Weighted uniform densities, J. Théor. Nombres Bordx., 19 (2007), 191-204.

[14] P. Kostyrko and T. Šalát, I-convergence, Real Anal. Exch., 26 (2000/2001) 669-686.

[15] H .I. Miller, A measure theoretical subsequence characterizaiton of statistical convergence, Trans. Amer. Math. Soc., 347 (1995), 1811-1819.

[16] H .I. Miller and C. Orhan, On almost convergence and statistically convergent subsequences, Acta. Math. Hungar., 93 (2001), 135-151.

[17] S. Pehlivan, Strongly almost convergent sequences defined by a modulus and uniformly statistical convergence, Soochow J. Math., 20 (1994), 205-211.

[18] E. Tas and T. Yurdakadim, Characterizaiton of uniform statistical convergence for double sequences, Miskolc Math. Notes, 13 (2012), 543-553.

[19] J. Zeager, Buck-type theorems for statistical convergence, Rad. Math., 9 (1999), 59-69.

(Received: November 20, 2015)

Tuğba Yurdakadim

Hitit University

Department of Mathematics

Çorum,Turkey

tugbayurdakadim@hotmail.com

Leila Miller-Wan Wieren

International University of Sarajevo

Faculty of Engineering and

Natural Sciences

71000, Sarajevo

Bosnia and Herzegovina

lmiller@ius.edu.ba 
